\title{
The Use of a Synthetic Ceramic Graft Mixed with Platelet-Rich Fibrin (PRF) in the Treatment of Bone Defects An experimental study
}

\author{
ALEXANDRU NEMTOI ${ }^{1}$, ANA NEMTOI ${ }^{2}$, KAMEL EARAR $^{3 *}$, ADRIAN BEZNEA $^{3 *}$, CRISTIAN ONISOR $^{3 *}$, DANISIA HABA $^{2}$ \\ ${ }^{1}$ Grigore T. Popa University of Medicine and Pharmacy, Department of Morpho-Functional Sciences, 16 Universitatii Str., 700115, Iasi, Romania \\ ${ }^{2}$ Grigore T. Popa University of Medicine and Pharmacy, Department of Oral and Maxillofacial Surgey, 16 Universitatii Str., 700115, Iasi, Romania \\ ${ }^{3}$ Dunarea de Jos University of Medicine and Pharmacy Faculty, Department of Dentistry, 47 Domneasca Str., 800008, Galati, Romania
}

The aim of this study is to investigate the effect of alloplatic graft and platelet rich fibrin (PRF), alone or in combination, on bone regeneration in Wistar rats femural defects. Bone defects were prepared in adults male of 20 Wistar rats. In a group of ten, the defect was filled with synthetic ceramic graft as an alloplastic graft; the others 10 were grafted with this alloplastic graft mixed with PRF. All animals were sacrificed on the $90^{\text {th }}$ postoperative day and the femural bones were removed, histologic sections were prepared and the experimental sites were examined microscopically. Histologic examination revealed more new bone formation in the defects filled with alloplastic graft mixed with PRF than in the defects grafted with alloplastic graft alone. The platelet rich fibrin combination effectively induces new bone formation.

Keywords: alloplastic material, graft, platelet-rich fibrin, histology

In the 1980s, the fibrin glue was the first blood-related product used in surgery. At that time, many authors then stated that it was used as a hemostatic agent and surgical glue, because of its positive effects on tissue healing [1]. In the following years, transforming growth factor $b$ (TGF- $\beta$ ) in the platelets were discovered, and many studies were performed for hard-soft tissue healing and regeneration [2]. In the 1990s, with the rapid development of the techniques and equipment, platelet-rich plasma (PRP), which contained a higher concentration of platelets than fibrin glue, was available. The first PRP study in the field of oral surgery was introduced by Whitman et al. in 1997 [3]. Nowadays, a number of different growth factors are used together with graft materials or by themself in maxillofacial and regenerative surgery. Among these is platelet-rich fibrin (PRF), which was developed by Dohan et al. [4]. With this simplified technique, an autologous fibrin matrix, which contains platelets and leucocyte growth factors, is obtained. PRF is a modification of PRP and is used in many medical specialties for tissue regeneration, as well as in oral and maxillofacial surgery. It is indicated for alveolar bone augmentation, sinus lift surgery, socket preservation after tooth extraction, defect reconstruction following cyst enucleation or tumor excision, and also alveolar cleft repair. This concentrate contains high levels of growth factors, including the platelet-derived growth factor (PDGF), tissue growth fcactor (TGF), vascular endothelial growth factor, insulin-like growth factor (IGF), and epidermal growth factor (EGF) [5]. These growth factors play a central role in hemostasis and the bone healing process, which makes PRF advantageous. Platelet growth factors are a well-known source of healing cytokines, usable for clinical applications. In many studies, PRF has a direct or indirect effects on bone regeneration in bone grafting or bone defect healing [6-8]. In the literature, authors have reported many advantages of PRF for bone regeneration [9]. There are also much controversies in the literature over the use of different grafts as bone substitutes [10]. The ideal biomaterials should provide osteoconductive and osteoinductive features similar to autogenous bone grafts, which are still considered the gold standard in reconstructive bone surgery. However, there is no ideal biomaterial. Although some studies have focused on the applications of PRF, few used animal models for experiments on bone regeneration with sole or combined applications of PRF and autogenous bone graft.

Alloplastic grafts may be made from hydroxyapatite, a naturally occurring mineral which is the main mineral component of bone, made from bioactive glass. Hydroxyapatite is a synthetic bone graft, which is the most used now due to its osteoconduction, hardness, and acceptability by bone. Some synthetic bone grafts are made of calcium carbonate, which start to decrease in usage because it is completely resorbable in short time and makes breaking of the bone easier. Finally used is the tricalcium phosphate in combination with hydroxyapatite and thus giving effect of both, osteoconduction and resorbability [11].

*email:erar_dr.kamel@yahoo.co; adrianbeznea@hotmail.com; cristi.onisor@gmail.com

All authors with equaly contributions 
The aim of the experimental study was to evaluate the efficiency of a single alloplastic graft and a mixed allograft with PRF on bone regeneration on femural bone defect in Wistar rats.

\section{Experimental part}

Animal model and PRF/ allograft protocol

For this experimental study, 20 male Wistar rats were acclimatized to the study conditions for a period of 14 days before starting the surgery protocol. All experimental designs and procedures have received approval of the Animal Ethics Committee of the "Grigore T. Popa" University of Medicine and Pharmacy, Iaşi, Romania. The animals were housed individually, at $25^{\circ} \mathrm{C}$. They were strictly pair-fed a laboratory diet containing $15 \%$ casein, $0.8 \%$ phosphorus, $1 \%$ calcium, 70-80\% carbohydrates, and 5\% fat throughout the experi- mental period. Demineralized water was available ad libitum. The surgical protocol foar each animal started with anesthesia which was initiated with Ketamine $\mathrm{HCl}$ (Ketavet ${ }^{\circledR}$, Ratiopharm, Ulm, Germany), followed by administration of inhalation anesthesia (Isoflurane $®$, Pharmacia \& Upjohn $\mathrm{GmbH}$, Erlangen, Germany). After general anesthesia, 6-8 $\mathrm{mL}$ of blood was obtained from the central artery of the ear. It was then transferred to plastic tubes (Vacuette, Greiner Bio-One, GmbH, Kremsmunster, Austria) and centrifuged for $12 \mathrm{~min}$ at $2700 \mathrm{rpm}$ (Hettich, Tuttlingen, Germany). After centrifugation, the blood separated into three layers. The middle layer, which represents PRF $(0.6-0.8 \mathrm{~mL})$, was taken (fig.1). A local anesthetic with articaine and epinephrine (Ubistesin ${ }^{\circledR} \mathrm{TM}$ forte, $3 \mathrm{M}$ Deutschland $\mathrm{GmbH}$, Germany) was also injected into the right femur of each animal, an incision was made at the distal metaphysis of the right femur and the bone was uncovered, where was perfomed a bone defect with a drill (fig.2 and fig.3). Ten animals received an alloplastic graft without PRF and 10 animal received a alloplastic graft mixed with PRF (fig.4) and then, according to the standard protocol, the periosteum and skin were sutured in two layers (Vicryl 5-0, Ethicon GmbH \& Co. KG, Norderstedt, Germany). The alloplastic graft used is a porous synthetic ceramic that contains $99 \%$ tricalcium phosphate (SyBone ${ }^{\circledR T C P}$, TAG Dental Israel). To reduce post-operative pain, Butorphanol $(0.05 \mathrm{mg} / \mathrm{kg}$ b.w.) was injected subcutaneously every 12 hours for five days. All animals received peri-operative antibiotics (Amoxiplus 1,2g) from one hour before surgery to seven days after surgery, to reduce the risk of infections.

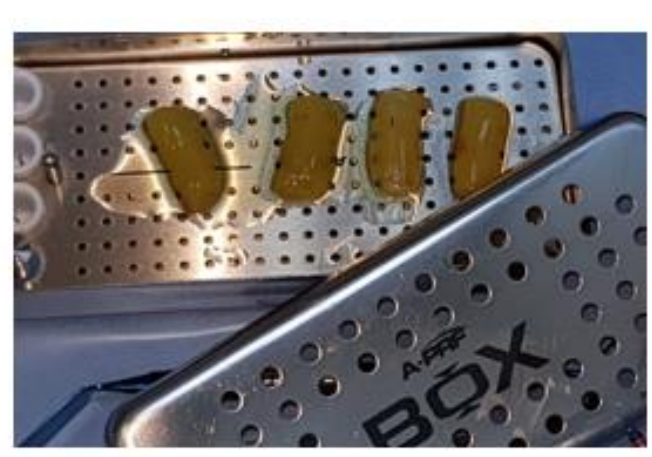

Fig. 1. The platelet-rich fibrin (PRF) prepared before combined with the allopastic graft

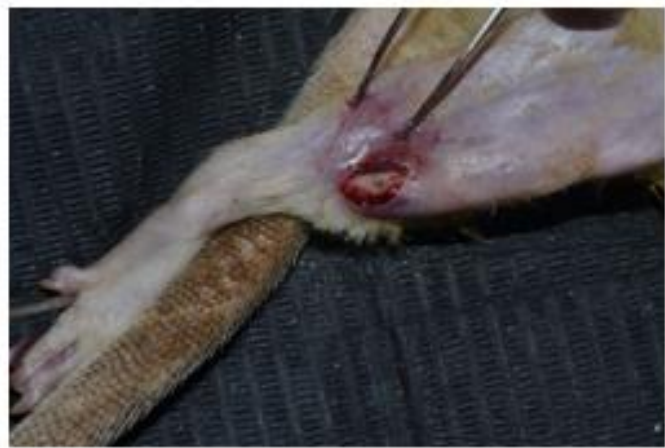

Fig. 3. The bone defect prepared before filling with single alloplastic graft and mixed alloplastic graft with PRF

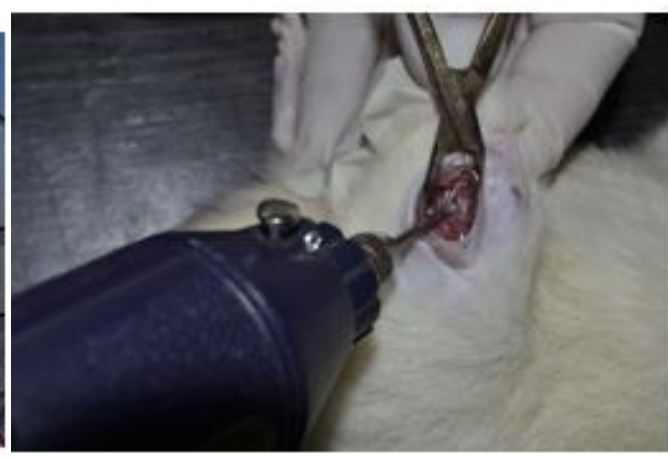

Fig. 2. The preparation of the bone defect in each right femural bone

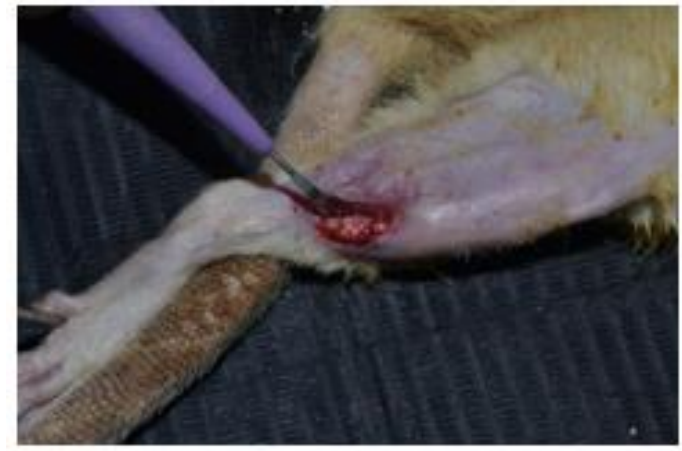

Fig. 4. The bone defect filled with alloplastic graft mixed with PRF 
At 90 days after surgery, animals were sacrificed. Each animal was sedated with an intramuscular injection of Ketamine (40 mg/kg b.w) and Midazolam ( $1 \mathrm{mg} / \mathrm{kg} \mathrm{b.w})$, followed by an intravascular injection of 20\% Pentobarbital solution into an ear vein until cardiac arrest occurred. The finding of absence of vital signs (respiratory movements, heartbeat, reflexes) animals will be dissected to harvest the right femur.

\section{Histological and histomorphometry analyses}

The bone samples were fixed in 10\% neutral buffered formalin (24-48 hours) and then decalcified in Bouin's solution $72 \%$. After tissue processing, the specimens were embedded into paraffin blocks (Leica TP1020, Leica Microsystems $\mathrm{GmbH}$, Germany). The paraffin blocks were cut into $5 \mu \mathrm{m}$ sections using Microtome SLEE CUT 6062 (SLEE Medical $\mathrm{GmbH}$, Germany). The sliced sections were deparaffinized and stained with Masson's trichrome techniques. Histomorphometry was performed using digital image analysis software (Leica Qwin Pro-image Leica Imaging Systems, Cambridge, UK). Five quantitative parameter were assessed: newly formed bone area, the quantity of residual allograft, connective tissue area, osteoblast and osteoclast numbers.

Statistics

All measurements were statistically evaluated using Statistical Package for the Social Sciences (SPSS) 19.0 for Windows (SPSS Inc., Chicago, IL, USA). Mean values and standard deviations (SDs) were calculated.

\section{Results and disscusions}

After the surgery, 1 animal died as a result of a fungal infection. During sacrifice, femural fractures were seen in 1 animal. The animal with fractures were excluded from the study. The remaining 18 animals recovered well from the surgical procedure, without any signs of inflammation or abscess formation.

Table 1 displays comparisons of the osteoblast and osteoclast number between Group A and Group B for all defects. Between Group A and Group B, there were difference was detected recording the number of osteoblast $(P<0.05)$.

Table 1

THE MEAN VALUE OF THE OSTEOCLAST AND OSTEOBLAST NUMBERS IN BOTH GROUPS

\begin{tabular}{|c|c|c|}
\hline Groups & Osteoblast number & Osteoclast number \\
\hline A. Single alloplastic graft & $25.51 \pm 2.3$ & $4.5 \pm 1.2$ \\
\hline B. Mixed alloplastic graft with PRF & $45.78 \pm 3.3$ & $3.7 \pm 1.8$ \\
\hline
\end{tabular}

There were no statistical differences in terms of the osteoclast number between the Group A and Group B. Table 2 presents a comparison of the newly formed bone area, residual material of alloplastic graft and conective tissue area of Group A and Group B for all defects. There were differences in the newly formed bone area between Group A and Group B $(P=0.0038)$. Also, there were differences in the residual bone graft and connective tissue area between Group A and Group B.

Table 2

THE MEAN VALUE OF THE NEW BONE AREA, RESIDUAL ALLOPLASTIC GRAFT AND CONNECTIVE TISSUE AREA IN BOTH GROUPS

\begin{tabular}{|l|c|c|c|}
\hline \multicolumn{1}{|c|}{ Groups } & New bone area & $\begin{array}{c}\text { Residual } \\
\text { alloplastic graft }\end{array}$ & $\begin{array}{c}\text { Connective } \\
\text { tissue area }\end{array}$ \\
\hline A. Single alloplastic graft & $76.7 \pm 2.3$ & $12.2 \pm 3.9$ & $11.1 \pm 3.4$ \\
\hline B. Mixed alloplastic graft with PRF & $93.3 \pm 1.9$ & $3.5 \pm 2.3$ & $3.2 \pm 1.8$ \\
\hline
\end{tabular}

At the level of the implantation area in the histological examination, in group A we can observe remodelling of the bone with presence of some bone defects filled with connective tissue and small parts of residual alloplastic graft (fig.5).

In the group B, a new formation of hawersian channels is observed, the concentration of concentric bone blades is delimited and we can observe much more cortical bone formed than in the group A (fig.6). 


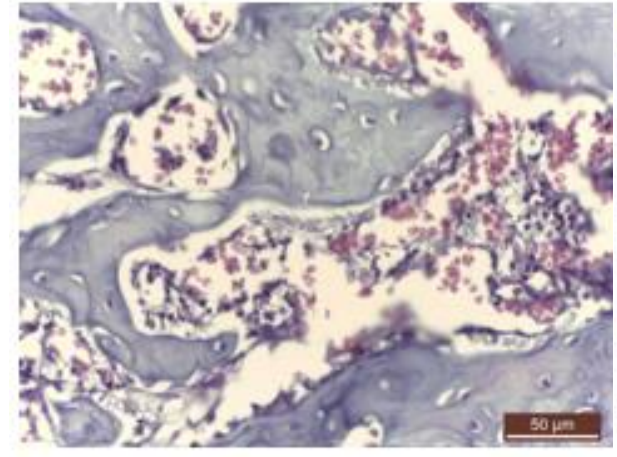

Fig. 5. Histological view of the bone defects in group A filled with single alloplastic graft

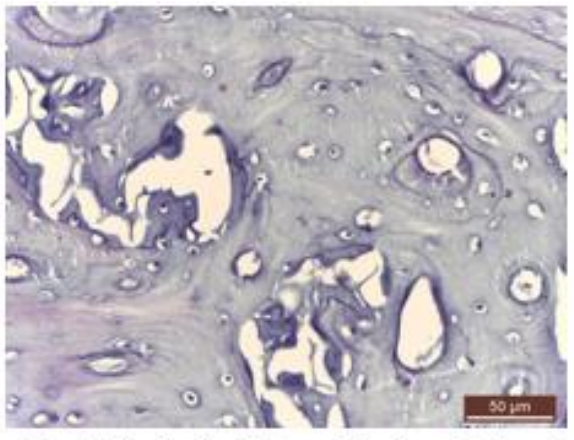

Fig. 6. Histological view of the bone defects in group B filled with mixed alloplastic graft with PRF

Results showed that when a combination of PRF and alloplastic graft was used for bone defects, the area of the new bone formed was significantly greater than in the group treated with these materials alone. In addition, histological findings showed that the quantity and maturation of the new bone formation was different in each group. This suggests that the efficiency of alloplastic grafts used can be improved by the addition of PRF.

Some authors compared autogenous grafts with autogenous grafts plus PRF for sinus lifting operations [12]. In the histomorphometric examination, the amount of the bone in autogenous graft /PRF combination group was more than in the group treated with autogenous bone grafts alone. In this situation, PRF activated the protein structure in the autogenous grafts and osteoblasts tended to adhere that were called into the environment area $[12,13]$.

Platelet-rich fibrin, as a fibrin biomaterial, carries the favorable constituents present in a blood sample such, as a large quantity of platelets and leukocyte cytokines [4]. Concentrated platelets contain many growth factors, including the PDGF, TGF- $\beta$, IGF, EGF, fibroblast growth factor, and bone morphogenic protein [5]. The role of platelets in the wound healing process has been extensively studied. These processes are mediated by various factors, including cytokines and growth factors that stimulate cell activity [14].

These growth factors play a central role in hemostasis, angiogenesis, osteoblastic proliferation and differentiation which makes PRF advantageous. Its molecular structure and low thrombin concentration are optimum for the migration of endothelial cells and fibroblasts [13]. PRF allows a significant postoperative protection of the surgical site and seems to accelerate the integration, maturation, and remodeling, while enhancing bone graft density [11].

Growth factors released from platelets, such as PDGF and TGF- $\beta 1$, have been shown to promote the regeneration of mucosa and bones $[15,16]$. PDGF has been reported to inhibit osteoblastic differentiation and stimulate cell proliferation in vitro. The effects of TGF- $\beta$ on osteoblastic differentiation depend on the dose and differentiation stage of the osteoblastic lineage [17].

In regeneration applications, rehabilitation of the bone defects requires more than a 3 month interval after bone grafting [18]. Thus, the growth factors that cause differences in osteogenic effects at early stages may have little or no effect in the long-term. Our study showed that defects for Group B showed more new bone formation than Groups A; hence, it can be inferred that applying growth factors to bone defects results in greater osteogenic effects than applying the alloplastic graft alone. The capacity of PRF to induce the growth of osteoblasts can help to increase the new bone regeneration when used in mixed with alloplastic grafts. Based on our results, applying PRF to the bone defects may accelerate the bone graft healing and shorten the time period for rehabilitation, even when we use alloplastic material for grafting.

\section{Conclusions}

The combination of platelet rich fibrin and allopastic graft, such as synthetic ceramic with $99 \%$ tricalcium phosphate can accelerate bone healing more than when these materials are used alone. In these situation, we can use alloplastic material with high results when we need more material to fill larger bone defects and we don't have enough autologous bone graft.

\section{References}

1.NIEKSCH, R., Zahn Mund Kieferheilkd Zentralbl., 68, 1980, p.555-561.

2.TAMIMI, F.M., TORRES, J., TRESGUERRES, I., CLEMENTE, C., LOPEZ-CABARCOS, E.,BLANCO, L.J., J Clin Periodontol., 33, 2006, p.922-928.

3.WHITMAN, D.H., BERRY, R.L., GREEN, D.M., J Oral Maxillofac Surg., 55, 1997, p.1294-1299.

4.DOHAN, D.M., CHOUKROUN, J., DISS, A., DOHAN, S.L., DOHAN, A.J., MOUHYI, J., Oral Surg Oral Med Oral Pathol Oral Radiol Endod., 101, 2006, p.45-50.

5.TOFFLER, M., TOSCANO, N., HOLTZCLAW, D., CORSO, M.D., DOHAN, D.M.. J Implant Adv Clin Dent., 1, 2009 , p.22-31.

6.KAIGLER, D., SILVA, E.A., MOONEY, D.J., J Periodontol., 84, 2013, p.230-238. 
7.PANDA, S., RAMAMOORTHI, S., JAYAKUMAR, N.D., SANKARI, M., VARGHESE, S.S., J Pharm Bioallied Sci., 6, 2014, p.127-131. 8.BARONE, A., RICCI, M., ROMANOS, G.E., TONELlO, P., ALFONSI, F., COVANI, U., Clin Oral Implants Res., 26, 2015, p.823-830. . 9.HE, L., LIN, Y., HU, X., ZHANG, Y., WU, H., Oral Surg Oral Med Oral Pathol Oral Radiol Endod.,108, 2009, p.707-713. 10.BILLSTRÖM, G.H., BLOM, A.W., LARSON, S., BESWICK, A.D., Injury., 44, 2013, p.28-33. 11.PRASANNA, K., BELLIAPPA, V.,, GHOUSIA, F., J Pharm Bioallied Sci., 5, 2013, p.125-127.

12.LEE, H.J., CHOI, B.H., JUNG, J.H., ZHU, S.J., LEE, S.H., HUH, J.Y., Oral Surg Oral Med Oral Pathol Oral Radiol Endod., 103, 2007, p.329333.

13.NESLIGÜL, N. K., TIMUCIN, B., YAVUZ, F.,Dental Research Journal., 12, 2015, p.418-424.

14.WERNER, S., GROSE, R., Physiol Rev 83, 2003, p.835-870.

15.MARX, R.E., CARLSON, E.R., EICHSTAEDT, R.M., Oral Surg Oral Med Oral Pathol Oral Radiol., 85, 1998, p.638-646.

16.MARX, R.E., J Oral Maxillofac Surg., 62, 2004, p.489- 496.

17.DINCER, Y., NECDET, D., AYDIN, O., METIN, S., BARIS, E.O., IBRAHIM, M., Acta Cir. Bras., 29, 2014, p.59-65.

18.PRAKASH S, THAKUR A., J Maxillofac Oral Surg., 10, 2011, p.45-49.

Manuscript received: 28.11 .2019 